\title{
RAPIDLY-CONVERGENT METHODS FOR EVALUATING ELLIPTIC INTEGRALS AND THETA AND ELLIPTIC FUNCTIONS
}

\author{
J. D. FENTON and R. S. GARDINER-GARDEN'
}

(Received 2 July 1980; revised 28 October 1981)

\begin{abstract}
The expressions for elliptic integrals, elliptic functions and theta functions given in standard reference books are slowly convergent as the parameter $m$ approaches unity, and in the limit do not converge. In this paper we use Jacobi's imaginary transformation to obtain alternative expressions which converge most rapidly in the limit as $m \rightarrow 1$. With the freedom to use the traditional formulae for $m \leqslant \frac{1}{2}$ and those obtained here for $m \geqslant \frac{1}{2}$, extraordinarily rapidly-convergent methods may be used for all values of $m$; no more than three terms of any series need be used to ensure eight-figure accuracy.
\end{abstract}

\section{Introduction}

The Jacobian elliptic functions $\operatorname{sn}(u \mid m), \operatorname{cn}(u \mid m), \operatorname{dn}(u \mid m)$, etc., where $u$ is the argument and $m$ the parameter, and the complete elliptic integrals $K(m)$ and $E(m)$ can be calculated in a number of ways. Methods include the use of power series, Fourier series, Landen transformations, and theta functions, for which various methods exist including the use of infinite series and products. Most of these are presented in the chapters by L. M. Milne-Thomson in [1]. These methods are not useful for all values of argument and parameter. For example, the power series are useful only for small arguments, and the Fourier series are not convergent if the parameter approaches unity. The Landen transformations are rapidly convergent, but are non-trivial to apply. If theta functions are used, the series and products for these are the most convenient of all for small values of

\footnotetext{
'School of Mathematics, University of New South Wales, Kensington, N.S.W. 2033.

(1) Copyright Australian Mathematical Society 1982
} 
the parameter. However, they too do not converge if the parameter approaches unity.

It is known that Jacobi's imaginary transformation may be simply applied to recast the expressions for theta functions so that they are most rapidly convergent in the limit as the parameter tends to unity. However, explicit presentations of these recast series seem not to have been given, except by Eagle [2, Section 3.53] who considered non-standard functions. The existence of these alternative expressions seems to be almost unknown.

In this paper, alternative series and products for theta functions are obtained using the imaginary transformation. These results are then used to give alternative expressions for the elliptic functions which also converge most rapidly in the limit where previously-presented expressions do not converge. Finally, alternative methods for the calculation of complete elliptic integrals are developed. These are shown to be the simple complement of well-known methods but, remarkably, seem to be unknown.

\section{Theta functions}

Theta functions are entire functions of the argument $z$, which also depend on a parameter $m$ which is usually in the range $0 \leqslant m \leqslant 1$. The zeros of the theta functions form an infinite rectangular lattice on the $z$ plane, while the functions themselves have a real period, and an imaginary pseudo-period which will be described below. There are several different definitions of the theta functions; initially the definition used here will be that used by Whittaker and Watson [4]. Subsequently these will be related to other definitions of the theta functions.

Consider the four functions defined by the Fourier series:

$$
\begin{aligned}
& \theta_{1}(z, q)=2 \sum_{n=0}^{\infty}(-1)^{n} q^{(n+1 / 2)^{2}} \sin [(n+1 / 2) 2 z] \\
& \theta_{2}(z, q)=2 \sum_{n=0}^{\infty} q^{(n+1 / 2)^{2}} \cos [(n+1 / 2) 2 z], \\
& \theta_{3}(z, q)=1+2 \sum_{n=1}^{\infty} q^{n^{2}} \cos 2 n z \\
& \theta_{4}(z, q)=1+2 \sum_{n=1}^{\infty}(-1)^{n} q^{n^{2}} \cos 2 n z
\end{aligned}
$$

where $q$ is the nome $q=q(m)=\exp \left(-\pi K^{\prime} / K\right)$, in which $K=K(m)$ is the complete elliptic integral of the first kind and where $K^{\prime}=K(1-m)$. The theta 
functions can also be obtained by infinite products, as given by [4, Section 21.3]:

$$
\begin{aligned}
& \theta_{1}(z, q)=2 G q^{1 / 4} \sin z \prod_{n=1}^{\infty}\left(1-2 q^{2 n} \cos 2 z+q^{4 n}\right), \\
& \theta_{2}(z, q)=2 G q^{1 / 4} \cos z \prod_{n=1}^{\infty}\left(1+2 q^{2 n} \cos 2 z+q^{4 n}\right), \\
& \theta_{3}(z, q)=G \prod_{n=1}^{\infty}\left(1+2 q^{2 n-1} \cos 2 z+q^{4 n-2}\right) \\
& \theta_{4}(z, q)=G \prod_{n=1}^{\infty}\left(1-2 q^{2 n-1} \cos 2 z+q^{4 n-2}\right)
\end{aligned}
$$

where $G$ is the infinite product

$$
G=\prod_{n=1}^{\infty}\left(1-q^{2 n}\right)
$$

From these it can be simply verified that the zeros of the functions form an infinite rectangular lattice as shown on Figure 1, the interval between zeros being $\pi$ horizontally and $\pi \tau$ vertically, where $\tau=i K^{\prime} / K$, such that $q=\exp (i \pi \tau)$.

The theta functions defined in (1) and (2) are singly-periodic with real periods. It can be shown that $\theta_{1}$ and $\theta_{2}$ have period $2 \pi$, while $\theta_{3}$ and $\theta_{4}$ have period $\pi$. By

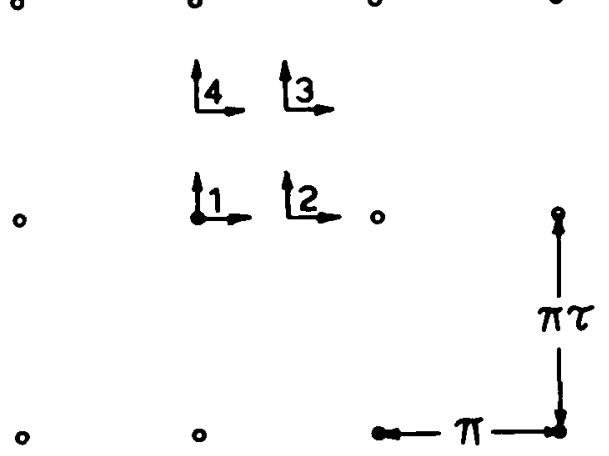

Figure 1. Part of complex plane, showing lattice of zeros. These zeros are those of the theta functions $\theta_{1}, \theta_{2}, \theta_{3}$ and $\theta_{4}$ if each function has the origin marked $1,2,3$ and 4 respectively. 
increasing $z$ in (1) by $\pi \tau$ it can be shown that the following pseudo-periodic relations hold:

$$
\begin{aligned}
& \theta_{1}(z+\pi \tau, q)=-q^{-1} e^{-i 2 z} \theta_{1}(z, q), \\
& \theta_{2}(z+\pi \tau, q)=q^{-1} e^{-i 2 z} \theta_{2}(z, q), \\
& \theta_{3}(z+\pi \tau, q)=q^{-1} e^{-i 2 z} \theta_{3}(z, q), \\
& \hat{\theta}_{4}(z+\pi \tau, q)=-q^{-i} e^{-i z z} \theta_{4}(z, q) .
\end{aligned}
$$

An important property is that

$$
\frac{d \theta_{1}}{d z}(0, q)=\theta_{2}(0, q) \theta_{3}(0, q) \theta_{4}(0, q),
$$

for which a rather lengthy proof is given in [4, Section 21.41].

\section{Jacobi's imaginary transformation}

The transformation is suggested by the rectangular array of zeros as shown in Figure 1 , for dividing $z$ by $\tau\left(=i K^{\prime} / K\right)$ is equivalent to a rotation of $-\pi / 2$ and a scaling of $|\tau|^{-1}$, so that the zeros of a theta function of argument $z / \tau$ would have a real interval of $\pi$, and an imaginary interval of $\pi / \tau$. This factor $1 / \tau$ is equal to $-i K / K^{\prime}$ and so the nome corresponding to an imaginary interval of $\pi / \tau$ is $q_{1}=\exp (i \pi / \tau)=\exp \left(-\pi K / K^{\prime}\right)$, which is precisely the complementary nome, $q_{1}(m)=q(1-m)$. From this outline it seems that two theta functions, one of argument $z$ and nome $q$, and the other of argument $z / \tau$ and nome $q_{1}$, have the same simple zeros and may be related to each other. That they are related and the form of this relationship can be established following the procedure of $[4$, Section $21.51]$, for the case of $\theta_{3}$, as follows.

It can be simply verified that the zeros of $\theta_{3}(z, q)$ and $\theta_{3}\left(z / \tau, q_{1}\right)$ are simple zeros at $z=\left(k+\frac{1}{2}\right) \pi+\left(n+\frac{1}{2}\right) \pi \tau$, where $k$ and $n$ are integers. The ratio of these two functions, denoted by $f(z)$, is an entire function with no zeros, hence the ratio

$$
\frac{f(z+\pi \tau)}{f(z)}=\frac{\theta_{3}\left(z / \tau+\pi, q_{1}\right)}{\theta_{3}(z+\pi \tau, q)} / \frac{\theta_{3}\left(z / \tau, q_{1}\right)}{\theta_{3}(z, q)}
$$

is also an entire function with no zeros. Rearranging, and using the fact that $\theta_{3}(z+\pi, q)=\theta_{3}(z, q)$ and the pseudo-periodíc relation (3.3), this gives

$$
f(z+\pi \tau) / f(z)=q e^{i 2 z}=\exp i(2 z+\pi \tau) .
$$

The quantity on the right can be expressed as $\exp i\left((z+\pi \tau)^{2} / \pi \tau\right) / \exp i\left(z^{2} / \pi \tau\right)$, and if $g(z)$ is introduced such that

$$
g(z)=f(z) \exp \left(z^{2} / i \pi \tau\right)
$$


then (5) gives $g(z+\pi \tau)=g(z)$, so that $g(z)$ has an imaginary period of $\pi \tau$. Similarly it can be shown that $g(z+\pi)=g(z)$, so that it also has a real period, of $\pi$. Thus, $g(z)$ is a doubly-periodic, entire function. As $\theta_{3}$ is bounded near the origin it can be shown that $g(z)$ is bounded near the origin. As it is doubly-periodic, it is bounded everywhere, and hence by Liouville's theorem, it is a constant, $g$ (for a particular value of $m$ ). Hence

$$
\theta_{3}(z, q)=g^{-1} \exp \left(z^{2} / i \pi \tau\right) \theta_{3}\left(z / \tau, q_{1}\right)
$$

and it may similarly be shown that

$$
\begin{aligned}
& \theta_{1}(z, q)=i g^{-1} \exp \left(z^{2} / i \pi \tau\right) \theta_{1}\left(z / \tau, q_{1}\right), \\
& \theta_{2}(z, q)=g^{-1} \exp \left(z^{2} / i \pi \tau\right) \theta_{4}\left(z / \tau, q_{1}\right),
\end{aligned}
$$

and

$$
\theta_{4}(z, q)=g^{-1} \exp \left(z^{2} / i \pi \tau\right) \theta_{2}\left(z / \tau, q_{1}\right)
$$

To determine $g,(6.1)$ may be differentiated, and substituting $z=0$ gives

$$
\theta_{1}^{\prime}(0, q)=i g^{-1} \tau^{-1} \theta_{1}^{\prime}\left(0, q_{1}\right)
$$

but use of (4) and (6) gives $g^{-2}=i \tau^{-1}, g= \pm\left(K / K^{\prime}\right)^{1 / 2}$, and considering (6.3) at $z=0$ it is clear that the positive sign should be taken.

The results as given by (6) are very useful, for values of the theta functions may be obtained from the transformed theta functions, which use the complementary nome $q_{1}$. As $q \rightarrow 1$, and the original series (1) become slowly convergent, then $q_{1} \rightarrow 0$, and the expressions on the right side of (6) are very rapidly convergent. Now, these alternative explicit expressions for the theta functions will be given.

\section{Alternative series and products}

Substituting the series (1) into the right side of (6) gives

$$
\begin{aligned}
& \theta_{1}(z, q)=2\left(K / K^{\prime}\right)^{1 / 2} \exp \left(-z^{2} K / K^{\prime} \pi\right) \sum_{n=0}^{\infty}(-1)^{n} q_{1}^{(n+1 / 2)^{2}} \sinh \left[\left(n+\frac{1}{2}\right) 2 z K / K^{\prime}\right] \\
& \theta_{2}(z, q)=\left(K / K^{\prime}\right)^{1 / 2} \exp \left(-z^{2} K / K^{\prime} \pi\right)\left\{1+2 \sum_{n=1}^{\infty}(-1)^{n} q_{1}^{n^{2}} \cosh \left(2 n z K / K^{\prime}\right)\right\} \\
& \theta_{3}(z, q)=\left(K / K^{\prime}\right)^{1 / 2} \exp \left(-z^{2} K / K^{\prime} \pi\right)\left\{1+2 \sum_{n=1}^{\infty} q_{1}^{n^{2}} \cosh \left(2 n z K / K^{\prime}\right)\right\}
\end{aligned}
$$


and

$\theta_{4}(z, q)=2\left(K / K^{\prime}\right)^{1 / 2} \exp \left(-z^{2} K / K^{\prime} \pi\right) \sum_{n=0}^{\infty} q_{1}^{(n+1 / 2)^{2}} \cosh \left[\left(n+\frac{1}{2}\right) 2 z K / K^{\prime}\right]$.

Whereas the nome appeared in the original series (1) as $q^{n^{2}}$, the complementary nome $q_{1}$ appears in these series as $q_{1}^{n^{2}}$. If $m<\frac{1}{2}$, the series (1) should be used, whereas if $m>\frac{1}{2}$, the scrics (7) are more rapidly convergent. Provided this boundary of $m=\frac{1}{2}$ is observed, the fortunate and powerful result is obtained that the expansion parameter used need never be greater than $q\left(\frac{1}{2}\right)=e^{-\pi}=0.04321$, which is raised to at least power $n^{2}$ in the $n$th term. These must be among the most rapidly convergent of all series as well as the simplest, for the coefficients in both (1) and (7) are either +1 or -1 in every case.

It is perhaps surprising that functions which are periodic in the real part of $z$ should be described by rapidly-convergent series of functions which are so clearly non-periodic as the hyperbolic functions. However, this is not much more remarkable than the fact that the series $x-x^{3} / 3 !+x^{5} / 5$ ! approximates a periodic function. In the use of the hyperbolic series (7) for arguments with a large real part, the hyperbolic functions may become large, rendering convergence slow. This is avoided if it is recognized that the functions $\theta_{1}$ and $\theta_{2}$ have a real period of $2 \pi$, while $\theta_{3}$ and $\theta_{4}$ have a real period of $\pi$. Hence

$$
\theta_{j}(z, q)=\theta_{j}(z(\bmod 2 \pi), q) \text { for } j=1,2,3,4,
$$

so that the actual value of $R(z)$ used in (7) can always be made to lie in the range $(-\pi, \pi)$. Thus the greatest magnitude of $\Omega(z)$ used in the calculations is $\pi$. For sufficiently large $z$ the hyperbolic functions in (7) all vary like $\exp \left(2 n z K / K^{\prime}\right)$, which on substituting the largest value $z=\pi$, becomes $q_{1}^{-2 n}$, and the individual terms in (7.2), for example, vary like $q_{1}^{n^{2}-2 n}$, so that successive terms are of relative magnitude $1, q_{1}, q_{1}^{4}, q_{1}^{9}, \ldots$. The last term need never be greater than $(0.04321)^{9} \approx .5 \times 10^{-12}$ : three terms are an excellent approximation!

If the Fourier series (1) are used when $z$ has a large imaginary part, then similar problems may occur. In this case the pseudo-periodic relations (3) can be used so that the series calculations need never be performed with an imaginary part of $z$ greater than $\pi \tau$ in magnitude, that is:

$$
\theta_{j}\left(z+\operatorname{ir} 2 K^{\prime} / K, q\right)=\left(q^{-2} e^{-i 4 z}\right)^{r} \theta_{j}(z, q) \text { for } j=1,2,3,4,
$$

where $r$ is any integer. Analysis similar to that for the hyperbolic series shows that successive terms vary like $1, q, q^{4}, q^{9}, \ldots$, so that three usually suffice.

Although the infinite-product expressions are not so rapidly-convergent as the series, the results of the imaginary transformation are here presented for completeness. Substituting the imaginary transforms (6) into the products (2) gives 


$$
\begin{aligned}
\theta_{1}(z, q)= & 2 q_{1}^{1 / 4}\left(K / K^{\prime}\right)^{1 / 2} G_{1} \exp \left(-z^{2} K / K^{\prime} \pi\right) \sinh \left(z K / K^{\prime}\right) \\
& \times \prod_{n=1}^{\infty}\left(1-2 q_{1}^{2 n} \cosh \left(2 z K / K^{\prime}\right)+q_{1}^{4 n}\right) \\
\theta_{2}(z, q)= & \left(K / K^{\prime}\right)^{1 / 2} G_{1} \exp \left(-z^{2} K / K^{\prime} \pi\right) \\
& \times \prod_{n=0}^{\infty}\left(1-2 q_{1}^{2 n+1} \cosh \left(2 z K / K^{\prime}\right)+q_{1}^{2(2 n+1)}\right) \\
\theta_{3}(z, q)= & \left(K / K^{\prime}\right)^{1 / 2} G_{1} \exp \left(-z^{2} K / K^{\prime} \pi\right) \\
& \times \prod_{n=0}^{\infty}\left(1+2 q_{1}^{2 n+1} \cosh \left(2 z K / K^{\prime}\right)+q_{1}^{2(2 n+1)}\right)
\end{aligned}
$$

and

$$
\begin{aligned}
\theta_{4}(z, q)= & 2 q_{1}^{1 / 4}\left(K / K^{\prime}\right)^{1 / 2} G_{1} \exp \left(-z^{2} K / K^{\prime} \pi\right) \cosh \left(z K / K^{\prime}\right) \\
& \times \prod_{n=1}^{\infty}\left(1+2 q_{1}^{2 n} \cosh \left(2 z K / K^{\prime}\right)+q_{1}^{4 n}\right),
\end{aligned}
$$

where $G_{1}=\prod_{n=1}^{\infty}\left(1-q_{1}^{2 n}\right)$. In the products (2) and (8) it can be seen that the nomes appear raised to the power $2 n$, whereas in the series (1) and (7) they are raised to the power $n^{2}$. Clearly the series converge more quickly, and are to be preferred in most applications.

\section{Neville's theta functions}

The theta function notation used by Neville (see [3] and [4, Section 16.36]) is of greater convenience in calculating elliptic functions, as will be seen in $\$ 6$ below. These four theta functions are $\theta_{s}, \theta_{c}, \theta_{d}$ and $\theta_{n}$, which are simply $\theta_{1}, \theta_{2}, \theta_{3}$ and $\theta_{4}$ respectively except that they are expressed as having $u=2 \mathrm{Kz} / \pi$ as argument (with spacings of zeros $2 K$ and $i 2 K^{\prime}$ ), dependence on the parameter is not shown explicitly, and they are scaled so that in the case of $\theta_{s}$ the derivative at zero is unity, while the other three have a function value of unity at zero. Thus,

$$
\begin{aligned}
& \theta_{s}(u)=2 K \theta_{1}(z, q) /\left(\pi \theta_{1}^{\prime}(0, q)\right), \\
& \theta_{c}(u)=\theta_{2}(z, q) / \theta_{2}(0, q), \\
& \theta_{d}(u)=\theta_{3}(z, q) / \theta_{3}(0, q)
\end{aligned}
$$

and

$$
\theta_{n}(u)=\theta_{4}(z, q) / \theta_{4}(0, q)
$$


It should be noted that the expression given in [1, Section 16.36.6] for $\theta_{s}$ is incorrect, the factor of $\pi$ in the denominator having been omitted. Now, use can be made of (4) and the formulae given in [1, Section 16.38] to give the relationships between the two sets of functions:

$$
\begin{aligned}
& \theta_{s}(u)=\left[\pi /\left(2 m^{1 / 2} m_{1}^{1 / 2} K\right)\right]^{1 / 2} \theta_{1}(z, q), \\
& \hat{\theta}_{c}(u)=\left[\pi / 2 m^{1 / 2} K\right]^{1 / 2} \theta_{2}(z, q), \\
& \theta_{d}(u)=[\pi / 2 K]^{1 / 2} \theta_{3}(z, q),
\end{aligned}
$$

and

$$
\theta_{n}(u)=\left[\pi / 2 m_{1}^{1 / 2} K\right]^{1 / 2} \theta_{4}(z, q),
$$

where $m_{1}=1-m$. For a given value of $m$, the quantities $m_{1}, K, K^{\prime}, q$ and $q^{\prime}$ may be calculated from expressions obtained in Section 7, then with $z=\pi u / 2 K$, either of the series (1) or (7) may be used to calculate the $\theta_{s}(u)$ etc., depending on whether $m \lessgtr \frac{1}{2}$.

\section{Jacobian elliptic functions}

These are doubly-periodic meromorphic functions, each of which may be defined as the ratio of any two of the theta functions $\theta_{s}, \theta_{c}, \theta_{d}$ and $\theta_{n}$, so that each elliptic function has the zeros of the theta function in the numerator and poles corresponding to the zeros of that in the denominator. The Jacobian elliptic function ab $u$ may be defined as

$$
\mathrm{ab} u=\mathrm{ab}(u \mid m)=\theta_{a}(u) / \theta_{b}(u),
$$

where $a$ and b may be any two of the letters $s, c, d$ and $n$ and where dependence on the parameter is shown by $m$ itself. The most commonly-encountered functions of the twelve possible are $\operatorname{sn} u, \operatorname{cn} u$ and $\operatorname{dn} u$, and it is possible to obtain any of the other nine from ratios of these. To obtain explicit formulae for calculating them, the formulae (9) may be used:

$$
\begin{aligned}
\operatorname{sn} u & =m^{-1 / 4} \theta_{1}(z, q) / \theta_{4}(z, q), \\
\operatorname{cn} u & =\left(m_{1} / m\right)^{1 / 4} \theta_{2}(z, q) / \theta_{4}(z, q), \\
\operatorname{dn} u & =m_{1}^{1 / 4} \theta_{3}(z, q) / \theta_{4}(z, q) .
\end{aligned}
$$

The series for these theta functions (1) or (7) may be substituted to give the following explicit formulae for the elliptic functions, where the infinite series have 
been replaced by the first three terms. If $m<\frac{1}{2}$ :

Use $z=\pi u / 2 K$ and

$$
\begin{aligned}
& \text { sn } u=2\left(\frac{q}{m}\right)^{1 / 4} \frac{\sin z-q^{2} \sin 3 z+q^{6} \sin 5 z-\cdots}{1-2 q \cos 2 z+2 q^{4} \cos 4 z-\cdots}, \\
& \text { cn } u=2\left(\frac{m_{1} q}{m}\right)^{1 / 4} \frac{\cos z+q^{2} \cos 3 z+q^{6} \cos 5 z+\cdots}{1-2 q \cos 2 z+2 q^{4} \cos 4 z-\cdots},
\end{aligned}
$$

and

$$
\operatorname{dn} u=m_{1}^{1 / 4} \frac{1+2 q \cos 2 z+2 q^{4} \cos 4 z+\cdots}{1-2 q \cos 2 z+2 q^{4} \cos 4 z-\cdots}
$$

If $m>\frac{1}{2}$ :

Use $w=\pi u / 2 K^{\prime}$ and

$$
\begin{aligned}
& \operatorname{sn} u=m^{-1 / 4} \frac{\sinh w-q_{1}^{2} \sinh 3 w+q_{1}^{6} \sinh 5 w-\cdots}{\cosh w+q_{1}^{2} \cosh 3 w+q_{1}^{6} \cosh 5 w+\cdots}, \\
& \operatorname{cn} u=\frac{1}{2}\left(\frac{m_{1}}{m q_{1}}\right)^{1 / 4} \frac{1-2 q_{1} \cosh 2 w+2 q_{1}^{4} \cosh 4 w-\cdots}{\cosh w+q_{1}^{2} \cosh 3 w+q_{1}^{6} \cosh 5 w+\cdots},
\end{aligned}
$$

and,

$$
\operatorname{dn} u=\frac{1}{2}\left(\frac{m_{1}}{q_{1}}\right)^{1 / 4} \frac{1+2 q_{1} \cosh 2 w+2 q_{1}^{4} \cosh 4 w+\cdots}{\cosh w+q_{1}^{2} \cosh 3 w+q_{1}^{6} \cosh 5 w+\cdots} .
$$

As each of these functions has a real period of $4 K$ and an imaginary period of $i 4 K^{\prime}$, no matter how large the value of $u$, it is a simple matter to bring the value of $u$ to be used in calculations to have a maximum real magnitude of $2 K$ and imaginary magnitude of $i 2 K^{\prime}$. If this is done, the series in the numerators and denominators are all rapidly convergent and for eight-figure accuracy may be truncated at the terms shown. The largest neglected terms are proportional to $q^{6}$ or $q_{1}^{6}$, which is never greater than $6 \times 10^{-9}$, provided the limitations in $m$ are observed. If greater accuracy is required, the series can easily be extended by inspection, where the general term of $q^{0}, q^{2}, q^{6} \ldots$ is $q^{n(n+1)}$, and of $q^{0}, q^{1}, q^{4}, \ldots$ is $q^{n^{2}}$.

From the expressions (10) and (11) the limiting values of the elliptic functions as $m$ approaches 0 or 1 can simply be obtained. Using [1, Section 17.3.28]:

$$
\lim _{m \rightarrow 0}\left(\frac{q}{m}\right)=\lim _{m \rightarrow 1}\left(\frac{q_{1}}{m_{1}}\right)=\frac{1}{16}
$$

and

$$
\lim _{m \rightarrow 0}(K)=\lim _{m \rightarrow 1}\left(K^{\prime}\right)=\frac{\pi}{2}
$$


gives: as $m \rightarrow 0$, sn $u \rightarrow \sin u, \operatorname{cn} u \rightarrow \cos u$ and $\operatorname{dn} u \rightarrow 1$, and as $m \rightarrow 1$, sn $u \rightarrow$ $\tanh u, \operatorname{cn} u \rightarrow \operatorname{sech} u$ and $\operatorname{dn} u \rightarrow \operatorname{sech} u$.

\section{The inversion problem: calculating elliptic integrals}

In many applications it is the parameter $m$ which is known initially whereas to appiy the series given in this work the elliptic integrals $K, K^{\prime}$ and the nome $q$ must be known. The calculation of these quantities is one of the classical problems in the field of elliptic functions-the inversion problem. There has never been a simple and explicit method for solving this over all values of $m$, despite the Landen transforms, and a useful series for small $m$ presented originally by Weierstrass [4, Section 21.8]. Unfortunately this series is not convergent in the limit $m \rightarrow 1$, which may explain its absence from [1], perhaps the most widely-used collection of results in this field.

Here, the alternative series developed in the present work will be used in an approach complementing that of Weierstrass so that $K$ can be calculated over all values of $m$ with rapidly-convergent series. A technique is used which is similar to that of Weierstrass, but using the complementary functions ( $q_{1}$ instead of $q$, and so on). From [4, Section 21.8]:

$$
m^{1 / 4}=\theta_{2}(0, q) / \theta_{3}(0, q),
$$

and substituting the $q_{1}$ expansions (7.2) and (7.3) gives

$$
m^{1 / 4}=\frac{1-2 q_{1}+2 q_{1}^{4}-\cdots}{1+2 q_{1}+2 q_{1}^{4}+\cdots} .
$$

If $q_{1}$ is known initially, this provides a convenient way of calculating $m$. In practice, $m$ is usually known, and this becomes a transcendental equation for $q_{1}$, which could be recast as a series for that quantity, however a more rapidly convergent procedure is obtained by introducing $\varepsilon_{1}$ :

$$
2 \varepsilon_{1}=\frac{1-m^{1 / 4}}{1+m^{1 / 4}}
$$

where $\varepsilon_{1} \rightarrow 0$ as $m \rightarrow 1$. Substituting for $m^{1 / 4}$ gives

$$
\begin{aligned}
2 \varepsilon_{1} & =\frac{\theta_{3}(0, q)-\theta_{2}(0, q)}{\theta_{3}(0, q)+\theta_{2}(0, q)} \\
& =2 \frac{q_{1}+q_{1}^{9}+q_{1}^{25}+\cdots}{1+2 q_{1}^{4}+2 q_{1}^{16}+\cdots},
\end{aligned}
$$


where the series in the numerator and denominator converge even more rapidly than those presented in Section 4. It is easily shown that this is equivalent to

$$
2 \varepsilon_{1}=\frac{\theta_{4}\left(0, q_{1}^{4}\right)}{\theta_{3}\left(0, q_{1}^{4}\right)}
$$

where the dependence on the parameter is in this case actually $q_{1}^{4}$. The object is, however, to obtain $q_{1}$ as a function of $\varepsilon_{1}$ and so (12) can be used to give

$$
q_{1}=\varepsilon_{1}+2 \varepsilon_{1}^{5}+15 \varepsilon_{1}^{9}+150 \varepsilon_{1}^{13}+O\left(\varepsilon_{1}^{17}\right) .
$$

An identical series exists for $q$ as a function of $\varepsilon=\frac{1}{2}\left(1-m_{1}^{1 / 4}\right) /\left(1+m_{1}^{1 / 4}\right)$, given in [4, Section 21.8], which is most suitable for $m \leqslant \frac{1}{2}$. Thus, as $m_{1}$ need never be taken greater than $\frac{1}{2}, \varepsilon_{1}$ is always less than 0.04321 , and for give-figure accuracy, only one term in (13) need be taken! It is interesting that this is the first series in this work where the coefficients are unable to be written down after inspection of the first few.

Having calculated $q_{1}, K^{\prime}$ is obtained using the result given in [1, Section 16.38.6]:

$$
K^{\prime}=(\pi / 2) \theta_{3}^{2}(0)=(\pi / 2)\left(1+2 q_{1}+2 q_{1}^{4}+2 q_{1}^{9}+\cdots\right)^{2},
$$

and $K$ obtained from the definition of $q_{1}$ :

$$
K=\left(K^{\prime} / \pi\right) \ln \left(1 / q_{1}\right) \text {. }
$$

Each of the results (13), (14) and (15) is simply the complementary form of those given by Weierstrass (see [4, Section 21.8]) but with $m, \varepsilon_{1}, q_{1}, K^{\prime}$ and $K$ replacing $m_{1}, \varepsilon, q, K$ and $K^{\prime}$ respectively. While this seems obvious in retrospect, it has not been shown or pointed out explicitly. For example in [4, Section 21.8] it is stated that the Weierstrass form can be used even for moderately large $m$, but without mentioning that it is useless as $m \rightarrow 1$ and without mentioning the existence of a complementary form better suited for $m \geqslant \frac{1}{2}$. For $m \leqslant \frac{1}{2}$ it is clearly advantageous to use the original Weierstrass form.

It is often necessary to calculate the complete elliptic integral of the second kind, $E(m)$, or its complement $E^{\prime}=E(1-m)$. An explicit method capable of high accuracy which uses quantities already calculated above is as follows. In [1, Section 17.3.23] a formula is presented:

$$
\frac{E}{K}=\frac{(2-m)}{3}+2\left(\frac{\pi}{K}\right)^{2}\left[\frac{1}{24}-\sum_{j=1}^{\infty} \frac{q^{2 j}}{\left(1-q^{2 j}\right)^{2}}\right],
$$

which is clearly useless if $m \rightarrow 1, q \rightarrow 1$. However, in Section 17.3.13 of the same book, Legendre's relation is presented:

$$
E K^{\prime}+E^{\prime} K-K K^{\prime}=\pi / 2 .
$$


If $m<\frac{1}{2}$, then $E$ can be found from (16) and subsequently $E^{\prime}$ from (17). Otherwise, $q_{1}$ and $K^{\prime}$ can be used in (16) to give $E^{\prime}$, and (17) used to obtain $E$.

\section{Acknowledgement}

The authors would like to thank Dr. A. N. Stokes and two referees for suggesting the use of Jacobi's imaginary transformation as a means of obtaining the equations (7), rather than using a longer method which we had described in a first draft of this paper.

\section{References}

[1] M. Abramowitz and I. A. Stegun, (eds.), Handbook of mathematical functions (Dover, New York, 1968).

[2] A. Eagle, The elliptic functions as they should be (Galloway and Porter, Cambridge, 1958).

[3] E. H. Neville, Jacobian elliptic functions (Oxford University Press, 1951).

[4] E. T. Whittaker and G. N. Watson, $A$ course of modern analysis (Cambridge University Press, 1952). 